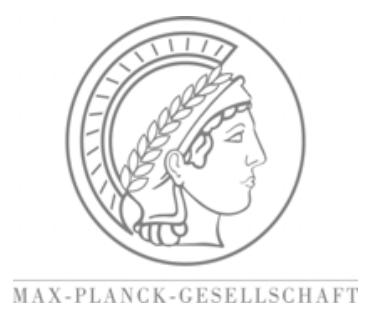

\# 0815

Symbolic Consumption and the Social Construction of Product Characteristics

by

Ulrich Witt

The Papers on Economics and Evolution are edited by the Evolutionary Economics Group, MPI Jena. For editorial correspondence, please contact: evopapers@econ.mpg.de

ISSN $1430-4716$

Max Planck Institute of Economics Evolutionary Economics Group Kahlaische Str. 10

07745 Jena, Germany

Fax: ++49-3641-686868

(C) by the author 


\title{
Symbolic Consumption and the Social Construction of Product Characteristics
}

\author{
Ulrich Witt*) \\ Max Planck Institute of Economics \\ Jena, Germany \\ witt@econ.mpg.de
}

\begin{abstract}
As recognized since long, consumption serving to signal social status, group membership, or self-esteem is a socially contingent activity. The corresponding expenditures are motivated mainly by the symbolic value they have for transmitting the signal. However, this presupposes some form of social coordination on what are valid, approved symbols. Unlike consumption not serving signaling purposes, the technological characteristics of the goods and services consumed may be secondary - what counts is their socially agreed capacity to function as a symbol. The paper discusses in detail the cognitive underpinnings of social agreement on consumption symbols and a model of their spontaneous emergence.
\end{abstract}

*) I owe thanks to Ori Heffetz for drawing my attention to the phenomenon of symbolic consumption and the participants of a workshop on the meaning of consumption at Johnson School, Cornell University, 2008 for helpful comments on an earlier draft of this paper. 


\section{Introduction}

In their historical growth process, modern economies have experienced dramatic changes in the size and composition of consumer expenditures on goods and services (see Lebergott 1993). Consumption has been transformed by rising real income, drifting relative prices, and innovations in product technology and product quality that have increased the variety of goods and services. Its many facets make the historical evolution of consumption a research topic that does not easily lend itself to an encompassing explanation. One crucial facet undoubtable is the qualitative change of consumer goods and services brought about by innovations. In his pioneering work on product variety as a feature of modern consumption, Paolo Saviotti has suggested a theory of technological evolution as a major explanatory element and has provided a powerful description of the changing product characteristics on this basis (Saviotti 1996; 2001; Saviotti and Metcalfe 1984, Saviotti and Trickett 1992, Nguyen, Saviotti, Trommetter and Bourgeois 2005).

However, important and appealing as the historical record of the objective, qualitative changes is, its explanation faces the old problem that it is the result of the choices of consumers who base their decisions not directly on technological characteristics and objective values. Whether innovative product variations succeed or fail and, hence, whether they are added to the existing consumption variety or not, rather depends on the consumers' subjective perceptions of, and preferences over, the alternatives that may also change over time. Being treated as a "black box" in subjective value theory, much less is known on the motives of the consumers" choices than on the technological characteristics of goods and services. It may be argued therefore that an improved understanding of the very changes in product technology and product variety (and the corresponding consumption expenditures) requires more elaborate hypotheses about consumer behavior (Windrum 2005).

In fact, as will be discussed in this paper, for a substantial part of consumption, the technological characteristics of the goods and services even appear to be of secondary importance for the consumers. To see why, it is necessary to dig somewhat deeper into the question of what motivates consumers to spend their money. Consumption (spending) is instrumental to help consumers satisfying their needs and wants. The motivation to consume thus arises from unsatisfied needs and wants. Their satisfaction can be improved more or less depending on the properties of the goods and services consumed. In many cases it can be conjectured that these properties correspond to the product characteristics. Yet, there are important exceptions. Consider the striving for social recognition and status seeking, for social identification, or for avoiding dissonances in one's self-perception. Where consumer expenditures are motivated in this way, consumption is obviously a socially contingent activity. This has been recognized since long (see Veblen 1898, Leibenstein 1950, Bourdieu 1984, Frank 1985, Moore 1994 for some significant examples).

More specifically, the attempt to signal status, group membership, or self-esteem by means of the consumption of certain goods and services rests on the symbolic value they have for transmitting the signal - it is "symbolic consumption" (see the work by Heffetz 2009). The distinct feature here is that the use of consumption symbols presupposes some form of social coordination on what are valid, approved symbols. In case of social identification and symbols of group membership the convention may result in the use of rather idiosyncratic, groupspecific, discriminatory consumption good or service. In case of status seeking and signaling for social recognition the convention may imply more widely recognizable (e.g. very expensive) 
consumption symbols. In any case, unlike consumption not motived to serve symbolic purposes, the technological characteristics of the goods and services consumed are secondary - what counts is their socially agreed capacity to function as a symbol. In a sense, thus, symbolic consumption is the form of consumer behavior least dependent on technological product characteristics. In the present paper it may therefore serve as a vantage point for emphasizing the role of motivational factors underlying consumption and its evolution over time.

The paper proceeds as follows. Drawing on earlier work (Witt 2001), Section 2 briefly outlines different motivations underlying consumer behavior - among them those of symbolic consumption - and puts them in perspective with the role that technological product characteristics can play for explaining the evolution of consumption. Section 3 turns to the cognitive mechanisms from which a socially shared understanding of consumption symbols can emerge. Section 4 presents a simple model of the actual coordination process by which a symbolic convention can spontaneously emerge on the basis of a socially shared understanding of the meaning of symbols. Section 5 highlights some implications of the model regarding the role of commercial advertising for both the emergence of a shared understanding of symbols and the actual collective coordination on the use of specific consumption symbols. Section 6 offers the conclusions.

\section{What Needs Does Symbolic Consumption Serve (and Why Does This Matter Anyway)?}

Consumption is a highly complex phenomenon. There is not only an enormous variety of goods and services to choose from, but usually also a multitude of motivations consumers pursue with their purchasing decisions. In the context of the present paper, it is useful to single out two different classes of motives by distinguishing certain properties which goods and services must have in order to be able to serve the different motives (for a detailed discussion see Witt 2001). A first class of consumption motives can be traced back to innate, physiological needs. Consumption activities directed at satisfying these needs typically show homoeostatic features, implying relatively stable satiation patterns when consumption possibilities are sufficiently abundant. Cases in point are the need to eat, to drink, to sleep, to maintain body temperature, etc.

In contrast, the second class of consumption motives arises from needs that are not physiologically determined, but that seem to be partly innate as well. Paradigmatic examples here are the needs for sensory arousal, for social recognition, or for consistency in one's selfperception. For different reasons, these needs and, hence, the motivation to consume which they trigger can lack the homoeostatic features of the physiological needs. They therefore tend to result in unstable satiation patterns when consumption possibilities become abundant. This can have dramatic economic and social consequence as was highlighted for the latter two needs in the work of, for example, Hirsch (1978) and Frank (1999).

Corresponding to the difference in the nature of the underlying needs, consumption technology differs too. The satisfaction of physiological needs depends on the physiologically defined intrinsic value of the products in terms of, e.g., content of calories, vitamins, minerals, flavor, etc. This is a case in which the technological product characteristics have obvious explanatory value for understanding consumption: it is for these intrinsic characteristics that the goods and services are consumed. Concerning the second class of motives, this is different. 
Intrinsic properties of products consumed to that end can contribute to need satisfaction at best indirectly, viz. to the extent to which they are conducive to signaling status to others or selfesteem to oneself. What really matters instead is the capacity of goods and services to serve as symbols for conspicuous consumption, conformist consumption, or non-conformist consumption, and this capacity is not intrinsic to the products. It results from a spontaneous process of tacit, social agreement among the members of a group or of society as to the consumption of what goods or services rather than others (with similar or the same intrinsic features) is accepted as a significant, relevant symbol.

It is well possible that some intrinsic characteristics - e.g. visibility or natural scarcity - increase the chances of some product to be accepted as a symbol. But the same intrinsic characteristics may be possessed by many more goods and services than those factually serving as accepted symbols. The argument to be elaborated further in this paper thus is that, because of the nature of the underlying needs that are served by it, symbolic consumption is to a large part a matter of social conventions: it is for their conventional - and to some extent arbitrary - status that certain goods and services are consumed as symbols conducive to social identification, status signaling, or self-esteem. The element of arbitrariness inherent to all conventions shows up in the (sub-) cultural contingency of symbolic consumption. What is appreciated in some (sub-) cultural contexts as a meaningful symbol does not necessarily qualify as such, or may even be counter-productive, in other contexts. This is true for conspicuous consumption meant to signal superior social status to other members of groups or societies, but even more so for signaling conformism or non-conformism (identifying with, or differentiating from, a group and its (sub-) culture). With increasing ability to spend on consumption, the tacit, social agreement on what qualifies as adequate symbols are likely to change so that the differentiating or conforming function of symbolic consumption is retained. This contributes further to the already mentioned unstable satiation patterns resulting in the fact that, in terms of their income elasticities, the corresponding goods and services qualify as luxuries. ${ }^{1}$

The distinction between the two classes of consumption motives and of the underlying needs not withstanding, goods and services consumed can serve needs from different classes simultaneously. The consumption of certain food and drinks or related services - to take an elementary example - can qualify as symbol of the kind just mentioned in addition to its functional value for the underlying physiological need. If so, the symbolic function of the product is influenced by, if it is not the outcome of, the formation of a tacit, social agreement that is largely independent of the intrinsic characteristics of food and drinks. Furthermore, if both kinds of motivation are present, income induced changes in actual spending behavior, i.e. the income elasticities of the corresponding goods and services, tend to hinge on the need(s) lacking homoeostatic satiation patterns - which are precisely those motivating symbolic consumption.

Accordingly, in the longer run it may be decisive for the income elasticities of goods and services whether or not their consumption is at least in part serving a symbolic function. To the extent to which this is the case, the social convention establishing the value as a symbol may be

\footnotetext{
Symbolic consumption that serves the purpose of assuring to oneself a certain self-perception (or of avoiding cognitive dissonances in this respect) is likely to be influenced not only by (sub-) cultural conventions, but also by the individual record of experience and learning. Therefore, consumption symbols that can factually be observed often also express subjective idiosyncracies that go far beyond the variety of (sub-) cultural standards.
} 
more important than the intrinsic properties of the goods and services. Hence, producers of goods and services who want to profit from the additional purchasing motivation (and a potentially higher income elasticity) have strong incentives to undertake promotion activities that target at influencing the process of social convention formation in a way favorable to their products. Under what condition this can be effective will have to be discussed below. Before, however, it is necessary to explain in more detail how the spontaneous processes work by which tacit, social agreement emerges and on what features of human behavior they rest.

\section{Symbolic Consumption and the Role of Social Cognitive Learning}

In the previous section it has been argued that symbolic consumption rests on spontaneously emerging conventions about what the goods and services are that function as symbols and what they mean. This presupposes that the individual agents share an understanding of what the messages conveyed by possible symbols are. Only on the basis of such a common understanding is it possible to coordinate on which symbols to use when. The fact that a socially shared understanding can emerge rests on a particular feature of human behavior. This feature, to be explained now in more detail, is the dynamic correlate of bounded rationality: the capacity to adapt by social cognitive learning. ${ }^{2}$

As a result of the limited cognitive resources, human attention, perception, and memory are highly selective. Furthermore, due to the fact that humans are social, communicating animals, their cognition is not entirely independent of that of other humans. This is due to the fact that, in groups with intense internal communication, the group members tend to tacitly develop similarities in their cognitive frames. As a result, there are also similarities within such groups concerning the question of where selective individual attention goes and what is selectively perceived and memorized. These similarities include the individual selection and understanding of possible coordination points (or "focal points", Sugden 1986). Indeed, this latter condition is an important key to understand how advertising activities can affect the spontaneous emergence of conventions in symbolic consumption. Advertising can suggest new consumption symbols that would otherwise not have gained attention or been considered. Relying on mass communication media it can align even large groups of agents in their tacit understanding of such possible new symbols.

To elaborate on the role of social cognitive learning, the interplay between attention processes, perception, and memory needs to be explained in more detail. We can draw here on findings from cognitive psychology (see, e.g., Anderson 2000, Chap. 3). Personal knowledge, knowledge about the meaning and context of symbolic actions being no exception, is acquired and modified over time through information that comes from own experience, from the observation of what others do, and from diverse forms of communication. However, individual information processing and, thus, knowledge acquisition are subject to significant constraints. In human perception a number of sensory stimuli, such as visual and acoustic signals, can be

Both concepts, that of bounded rationality (Simon 1972) and that of social cognitive learning (Bandura 1986), rest on the same view of limited human cognitive resources constraining human information processing and decision making. Yet social cognitive learning theory emphasizes the learning dynamics and the social influences on what is learnt that tend to be neglected in the decisiontheoretic framework of the literature on bounded rationality. 
recognized in parallel. Yet, unless more lasting attention is paid to any such signal it will quickly escape from memory. Hence, there is a kind of filter determining what specific stimuli or signals are processed further and what not. This filter is provided by selective attention processes: only signals gaining attention are processed further in thinking and memorizing. ${ }^{3}$

What signals gain attention depends on both their physically based and their meaningbased attributes. Concerning the physically based attributes, it has been shown that the relevant variables are frequency and relative strength or intensity of a stimulus (Helson 1964) - in the present context the frequency of exposure to a particular signal and the sensory quality by which it is presented (e.g., loudness or visibility). Regarding the meaning-based attributes, the attention paid to a signal depends on whether the cognitive system can attribute a meaning to it and what emotional value the meaning has (Anderson 2000, Chaps. 6 and 7). A meaning is identified through tracing information from long term memory. Long term memory is the store of individually accumulated knowledge. In order for elements of long term memory to be made available, they have to be activated selectively through cognitive cues contained in incoming signals. Hence, to be given a meaningful interpretation, signals must contain cues to which information stored in long term memory can be associated.

A particular case in point is information associated with a meaning that has emotional connotations. Translated (in an admittedly incomplete form) into the categories of liking or disliking of the economic model of behavior, the emotional value of an information can be interpreted as relating to individual tastes. By extension of the previous hypotheses the following may then be concluded. If an individual has developed a liking or disliking for some item, incoming signals that can be related to that item have a more important meaning than those that cannot. Hence, they, and the information they contain, attract more attention. Since the importance attributed to some information is highly correlated with the frequency with which that information is attended and rehearsed, such information tends to also be more prominently represented in long term memory, i.e. personal knowledge.

It has to be noted, though, that despite its stunning capacity long term memory is far from being perfect. Information stored in long term memory is retrieved by cognitive traces (see Anderson 2000, chap.7). To forget a particular piece of information stored earlier in long term memory amounts to a loss of retrieval of that information due to insufficient practicing of the memory traces and their subsequent decay. The crucial point is that because of the limited information processing capacity of the working memory, practicing some particular memory traces rather than others is always at the expense of those other memory traces. Therefore, an information that has less frequently and the less intensively been recalled in the past is likely to be lost from long term memory, i.e. from current knowledge. Accordingly, frequency (of practicing those traces) and intensity (of the liking/disliking of the stored information) are once more the decisive variables, this time with regard to the probability and speed of recalling information.

\footnotetext{
Put in economic terms, this implies that the attention given to stimuli coming from the environment is a scarce cognitive resource that has alternative uses. This insight has been interpreted to imply an allocation problem for which a rational solution should exist (see Radner and Rothschild 1975). As far as the attention processes of the sensory system are concerned, however, their working and the resulting allocation of attention are not subject to deliberate choice and planning. Attention processes are spontaneously triggered. They select by their own rules what relatively small subset of stimuli often abundantly offered to the sensory system from the environment actually reaches the working memory.
} 
In view of the constraints on human perception and memory, it is not possible for the individual consumer to know, and keep track of, all the huge variety of both goods and services in modern economies and possible symbolic content implied by their consumption. At any point in time consumers can only hold knowledge about fractions of the entire consumption possibilities. What is more, as a result of the self-augmenting interplay between attention processes, selective perception, and memory retrieval, consumers tend to specialize and develop an increasing sophistication in certain consumer knowledge and the corresponding symbolic and non-symbolic consumption activities in the following sense:

(i) Since attention shifts from information less frequently and less intensely recognized towards information recognized more often and more intensely, current knowledge and tastes shift over time to those consumption possibilities and symbols that gain more attention (shift effect);

(ii) the perception and knowledge of some particular consumption possibilities and symbols that continue to attract attention tend to become more refined over time, and tastes tend to be developed for more detailed attributes of these possibilities and symbols (refinement effect).

A question that remains is what determines the frequency and intensity of signals that can, or cannot, possibly gain attention. Apart from the already mentioned physically based and meaning-based factors influencing the intensity, is this an entirely randomly determined process or are there any systematic features? It is at this point that the role of social-cognitive learning becomes relevant. The individual selective information processing and learning just outlined does not take place in an entirely isolated, autonomous way. Rather, it is molded in interactive processes with the individuals' social environment (Bandura 1986, Chap. 2). Communication with, and observation of, other agents are a prominent source of information, a major factor in attracting attention, and an important instance of learning.

This is particularly relevant for consumption activities. The more intense and lasting communication about, and observational learning on, the consumption behavior of other individuals in the same social environment - a group of peers, say - are, the more likely the individuals involved experience parallel shift and refinement effects in their perception and knowledge of consumption. They tend to develop collectively shared interpretation patterns and a common knowledge of symbols and symbolic practices - a first step to tacit agreement on which ones to use when. The consequence is a significantly reduced variety of symbols and symbolic practices that is characteristic for the sub-culture of such groups. (In part, these cognitive commonalities result from the fact that, in intensely communicating groups, the agents' selective information processing is occupied with much the same topics which are processed in parallel leaving less attention for other topics. In part, collectively shared interpretative frames emerge in an intuitive way from mutual observational learning.)

Observational learning is also behind the formation of "social models" (Bandura 1986, Chap. 7) of consumption standards ('keeping up with the Johnsons') and symbolic practices ('doing like the Johnsons do') which, when commonly accepted, imply a tacit agreement on some conventional form rather than a multitude of possible others. As a result of intense communicating within groups, the group members focus on much the same limited set of consumption patterns and symbolic practices. To the extent to which they are recognized as specific to the group, they become models of behavior - often with the normative connotation of a behavior expected to be shown by group members. 
In groups with intense internal interactions, behavior deviating from the social model in the present context the use of consumption symbols other than the conventional ones - will not only be observed by the other group members. Together with its conjectured motives it is likely to also be made an object communication and group-internal opinion formation processes. Such a deviation means that actions not previously considered by the other group members are brought to their attention. They observe what, in a sense, is a vicarious experiment with uncertain outcome that saves them own efforts or costs in conducting such experiments. If the consequences of the experiment are assessed as rewarding, the other group member are likely to sooner or later imitate the deviating behavior. Rewards to deviating behavior can come in different forms. In case of symbols expressing conformism, the reward can result from having found a more convenient or less costly form of symbolic consumption to signal conformism. In the case of status signaling conspicuous consumption, in contrast, acquiring and enjoying the possession of something more expensive and therefore usually more scarce and harder to get may be experienced as a reward. In the case of symbolic consumption signaling nonconformism, to reach distinction by creating unusual or even provocative forms of consumption may cause the desired, rewarding effect.

As a consequence of the possible deviations of some group members, social models of symbolic consumption and the underlying socially shared tacit agreements on what the relevant symbols are and what they mean are not necessarily stable. Vicarious experiments with symbols are a permanent threat to the conventional basis of symbolic consumption, but they are not all successful. In fact, the stability of conventions in symbolic consumption can be expected to depend on the frequency of deviating behavior, on the assessment of its consequences by the other group members, and on the openness of the group members to such experiments that may be influenced by advertising campaigns on the part of the producers of consumption goods and services. These conditions and the dynamics they imply require a more detailed discussion in the next section.

\section{Emergence and Decline of Conventions about Consumption Symbols}

It has been claimed above that the spontaneous emergence of the social conventions in which symbolic consumption activities are molded requires a shared understanding of the relevant symbols and their meaning. However, for the actual establishing of conventional symbols it is required in addition that the agents' consumption activities do coordinate on, and keep to, the use of these symbols. This is not automatically following from socially sharing knowledge about the meaning of symbols.

In the previous section, the first prerequisite has been explained by the limitation of the human cognitive system that results in its high selectivity and by the social embeddedness of selective learning processes. Even though the tacit agreement on symbolic consumption that can spontaneously emerge within groups with intense internal communication is likely to shape the perception of what choices to make in what situation, there may be behavior that more or less deliberately deviates from the conventional use of symbols. This confronts the other group members with alternatives not previously considered so that they now face a choice of whether or not to imitate the deviation. This choice directly affects the second precondition for the emergence of conventions in symbolic consumption: the coordination on symbolic action in terms of the actual spending behavior. A simple model can help to clarify what the implications 
are for the stability of the conventional basis of symbolic consumption. To keep things simple we will confine us here to the case of consumption symbols expressing conformism. ${ }^{4}$

As all conventions, those determining what symbol to use in what situation draw their strength from the fact that the message implied by symbolic consumption (in this case to use the group symbols to demonstrate identification with the group) is the more easily and the less ambiguously conveyed the more widely the convention is used in corresponding consumption activities of a group or of society. Let us therefore denote the fraction of group members who at some time $t$ try a deviation from the hitherto conventional symbolic consumption activities by $\mathrm{F}(\mathrm{t}), 0 \leq \mathrm{F}(\mathrm{t}) \leq 1$. (The share of group members sticking to the original symbols is then given 1 $\mathrm{F}(\mathrm{t})$ ). Assume that initially $\mathrm{F}(\mathrm{t})=0$, until somebody in the group deviates from that convention and that this happens for some good reason. The deviation is likely to attracts the attention of the other group members who become aware of a new action possibility and engage in an interactive opinion formation process in which they try to assess the motives and outcomes of the deviation. They then have to choose whether or not to imitate the deviation.

Let $\mathrm{p}(\mathrm{t})$ be the probability that at time $\mathrm{t}$ another group member imitates the deviation, because she assesses the consequences of deviating as sufficiently rewarding to compensate the likely ambiguity arising from using unconventional consumption symbols. $p(t)$ thus reflects an assessment of motives and outcome of the vicarious experiment. Several factors play a role for this assessment. First, the ambiguity resulting from using symbols other than the hitherto valid ones always curtails the potential reward of deviators. However, since the ambiguity is rapidly decreasing with a rising fraction of deviators (i.e. with a rising value of $\mathrm{F}(\mathrm{t})$ ), it can be stated:

Assumption 1 The probability $\mathrm{p}(\mathrm{t})$ that at time $\mathrm{t}$ a group member imitates the deviation increases more than proportionately with increasing $\mathrm{F}(\mathrm{t})$.

Second, the assessment is heavily dependent on how the possible benefits from deviating, i.e. from changing to other consumption symbols, are perceived. As explained in the previous section, this in turn, hinges on the group members' current state of likings and knowledge. Established forms of symbolic consumption are likely to be the result of some kind of specialization in consumption by the group members that has already taken place. Deviations in the direction of further refinements of consumption may therefore meet less, but still enough, reservations than shifts to consuming different goods and services for which different consumption knowledge would have to be acquired and learned likings would have to be devaluated. However, such reservations and the natural conservatism that conformist symbolic consumption implies can be overcome by in opinion formation processes provided suitable information is powerfully put on the group's agenda. This does not necessarily have to come from inside the group (e.g. by opinion leaders). Indeed, the fact that the group's agenda can be influenced from outside the group draws attention to a third factor, the role of the media in influencing the beliefs and likings of the group members.

\footnotetext{
This means that the model to be discussed represents a variant of the band-wagon effect already described by Veblen (1899). This effect belongs, in turn, to the class of frequency-dependency effects which lend themselves to a particularly simple analysis when a bi-modal case can be assumed as in the choice between sticking to an established convention or choosing a new one. The model presupposes that a group of consumers can be defined who communicate with one another in a sufficiently intense form to make social-cognitive learning possible.
} 
Assumption 2 Conservatism partly due to earlier specialization in consumption can drive the probability $\mathrm{p}(\mathrm{t})$ to zero unless, in the opinion formation process of the group, attention can be directed at information suitably propagating the deviation.

The effect postulated by assumption 2 can be captured in the model by two elements. One is a properly calibrated bias factor $1+b(t)$ that lowers or raises $p(t)$. The other element is the variable $b(t)$ in the bias factor. It will be made dependent here on the cumulative effort $x(t)$ $\geq 0$ that is put into placing favorable information on the group agenda and a measure $\lambda, 0 \leq \lambda$ $\leq 1$, for the efficacy of that information as follows:

(1) $\quad b(t)=1-2^{(1-\lambda x(t))}$.

According to eq. (1), $\mathrm{b}(\mathrm{t})$ goes from -1 to +1 if $\mathrm{x}(\mathrm{t})$ is sufficiently raised, provided $\lambda>0$. Hence, with insufficient effort (sufficient effort) to place favorable information and/or with insufficient efficacy (insufficient efficacy) of the placed information - a bias contra (pro) changing to new forms of symbolic consumption is assumed. If assumption 1 is accounted for by means of a simple quadratic specification of the dependence of $q(\mathrm{t})$ on $\mathrm{F}(\mathrm{t})$, taken together with assumption 2 it results in

$$
\mathrm{p}(\mathrm{t})=(1+\mathrm{b}(\mathrm{t}))[\mathrm{F}(\mathrm{t})]^{2} .
$$

Turning now to a group member who has tried the deviation, the experienced outcome may support the experiment undertaken or induce a switching back to the original convention. Let the probability for a deviator to switch back at time $t$ be given by $q(\mathrm{t})$. Analogously to discussion of the factors that influence $\mathrm{p}(\mathrm{t})$, it can be argued that

Assumption $3 \mathrm{q}(\mathrm{t})$ increases more than proportionately with an increasing value of $1-\mathrm{F}(\mathrm{t})$ and is inversely affected by the variable $b(t)$, i.e. subject to a bias factor $1-b(t)$.

Hence, when a quadratic specification is chosen again,

$$
\mathrm{q}(\mathrm{t})=(1-\mathrm{b}(\mathrm{t}))[(1-\mathrm{F}(\mathrm{t}))]^{2} \text {. }
$$

The dynamics of the convention underlying symbolic consumption in the case of conformist symbols is captured by the change over time of the fraction of group members who deviate from the hitherto conventional symbols. In discrete time this can be expressed by the first order difference equation

$$
F(t+1)=\left\{\begin{array}{l}
0 \quad \text { for } F(t)<0 \\
F(t)+(1-F(t)) p(t)(1+b(t))-F(t) q(t)(1-b(t)) \\
1 \text { for } F(t)>1
\end{array} \quad \text { for } F \in[0,1]\right.
$$

After inserting eqs. (2) and (3) into (4) and some rearranging we get the cubic equation

$$
\mathrm{F}(\mathrm{t}+1)=\mathrm{b}(\mathrm{t}) \mathrm{F}(\mathrm{t})+(3-\mathrm{b}(\mathrm{t}))[\mathrm{F}(\mathrm{t})]^{2}-2[\mathrm{~F}(\mathrm{t})]^{3}
$$

and can state 
Proposition Given assumptions 1 - 3 and the corresponding specifications in eqs. (1) - (3) the attractors of eq. (5) bifurcate when $b(t)$ grows from -1 to +1 . At the same time $\mathrm{F}(\mathrm{t})$ goes from 0 to 1 .

Since the bifurcation is a well known feature of first-order cubic difference equations (see e.g. Lorenz 1993, Chap. 3), a proof of the proposition can be omitted here. Instead, the bifurcation is graphically displayed in Figure 1.

The role of the bias variable $b(t)$ in triggering the bifurcation is as follows. For $b(t)=-$ 1 the attractor $F^{*}=0$ is globally stable. If $x(t)$ is raised and for given $\lambda$ so that $b(t)$ grows, another attractor occurs in $\mathrm{F}^{* *}=1$. The bifurcation implies changing basins of attraction of $\mathrm{F}^{*}$ and $\mathrm{F}^{* *}$ as $\mathrm{b}(\mathrm{t})$ grows further. If two locally stable attractors exist simultaneously in the interval $[0,1]$, their respective basin of attraction is separated by an unstable fixed point $\mathrm{F}^{\circ}$. As can be seen from Figure 1 , for $b(t)=-1, F_{(1)}^{\circ}=1$; the entire interval $[0,1]$ is the basin of attraction for $\mathrm{F}^{*}$. Upon further growth of $\mathrm{x}(\mathrm{t})$ and $\mathrm{b}(\mathrm{t}) \mathrm{F}^{\circ}$ moves down the $45^{\circ}$-degree line into $\mathrm{F}_{(2)}^{\circ}$ and further on into $\mathrm{F}^{\circ}{ }_{(3)}$. For $\mathrm{b}(\mathrm{t})=1, \mathrm{~F}^{\circ}$ converges to 0 . The attractor $\mathrm{F}^{*}$ vanishes and eventually gives way to a globally stable attractor $\mathrm{F}^{* *}$ with $[0,1]$ as the entire basin of attraction. ${ }^{5}$

Figure 1 about here

Figure 1 Bifurcation of Attractors in the Transition Between Conventions about Symbols

The above Proposition has two implications that are important for understanding the influence that the particularities of symbolic consumption have on the evolution of consumption more broadly. First, since the technological characteristics of the corresponding products are secondary in importance, goods and services incorporating technological innovations that improve those characteristics do not necessarily enjoy a greater probability of being adopted as the valid symbol in the collective convention formation process. Nor are they necessarily more likely to replace existing consumption symbols that are technically inferior unless their superiority causes the bias parameter to increase. To the extent to which consumption serves symbolic purposes, the technological evolution in the product space therefore has only an indirect, if any, effect on changes in consumption behavior. Second, any effort to overcome the natural conservatism in learned consumption behavior by placing information that is favorable for deviating from received forms of symbolic consumption must be able to attract massive attention in order to be able to drive up the bias parameter and thus make a transition to a convention on a new symbols possible. The efforts may be undertaken either from inside the group or from outside. Those coming from outside may be considered the more interesting and will therefore be paid closer attention to in the next section.

\footnotetext{
Note that the first order difference eq. (5) and the corresponding graphs in Figure 1 represent mean processes. In the realization of the stochastic switching process based on the probabilities $p(t)$ and $\mathrm{q}(\mathrm{t})$ the actual increment $\Delta \mathrm{F}(\mathrm{t}) / \Delta \mathrm{t}$ fluctuates around the expected one given by (5). Consequently, the closer $\mathrm{F}^{\circ}$ approaches 0 , the greater the chance that by a cumulation of random fluctuations $\mathrm{F}(\mathrm{t})$ is pushed beyond $\mathrm{F}^{\circ}$ and the process then is attracted to $\mathrm{F}^{* *}=1$. For a detailed discussion of the relationship between stochastic realizations and the mean process see Weidlich (2000).
} 


\section{Advertising, Agenda Effects and Symbolic Consumption}

The selective attention processes that have been argued above to play a key role in the emergence of socially shared similarities in perception, tastes, and knowledge tend to discriminate against entirely novel information. They thus stabilize and preserve the cognitive prerequisites for the common understanding of what consumption symbols mean. Individual perceptions, tastes, and knowledge may become refined over time, and these refinements may be socially shared in a group. (In fact, larger groups may increasingly differentiate into subgroups by different, selective refinement in consumption.) However, this does not change the conservative tendency of the processes.

Something similar holds for the collective coordination process that results in a tacit agreement (a convention) about what symbols to consume under what conditions. As the model of conformist symbolic consumption in the previous section showed, hitherto conventional symbols tend to resist attempts to deviate and to shift to new symbols, simply because there either exists no stable attractor $\mathrm{F}^{* *}$ representing collective coordination on alternative symbols or, if it exists, because it is almost certain that no spontaneous transition to $\mathrm{F}^{* *}$ passing beyond the critical fraction of deviators $\mathrm{F}^{\circ}$ will occur. Any deviation experiment that occurs and that may temporarily find a few imitators is almost surely given up sooner or later so that $\mathrm{F}(\mathrm{t})$ returns to 0. (As was explained, the only exception is a massive and effective placement of information on advantages of the new consumption symbols that could drive the bias variable from - 1 to +1$.

The question then is what these conservative tendencies imply for the influence that possible commercial promotional activities and advertising by producers can have on symbolic consumption. Under what condition, if at all, do they affect either what goods and services are used as symbols or the volume of expenditures on certain forms of symbolic consumption? In the light of the discussion of the consumers' cognitive capacity, for a manipulation of symbolic consumption activities to work it would be necessary that the corresponding advertised information is intense and frequent enough to be noted by the consumers against a background of a huge amount of other information competing for individual attention. This is not easy to achieve. If it would appear to be possible to manipulate consumers spending behavior, this would most likely elicit all sorts of endeavors to do so - with the outcome of a competitive race for the consumers' attention. In this competition, the impact each single advertisement can have on the consumers' attention allocation would, however, be eroded.

Once a competitive race is started, the effect of each single, costly advertised piece of information on average tends to be marginalized (the possibility that a few of them gain more attention not being excluded). Yet, not emitting information at all while the competitors do means to lose even the average marginal attention. Under such conditions a huge amount of competing advertisement is likely to be elicited with the effect that a direct manipulation of the information factually processed by the consumers is unlikely to happen. Yet, there may be indirect effects amounting to a kind of unplanned, collectively achieved manipulation of the consumers' perceptions. While it may be difficult to gain attention for information relating to the specific products of a particular advertising producer, the advertisement of a whole industry may jointly contribute to the emergence and stabilization of certain consumption symbols.

If, for instance, the industry's advertisements equally appeal to status recognition as a prominent features, this may foster awareness of social status considerations and receptiveness 
to status signals among the consumers on which the industry's advertisement is targeted. Hence, to give an example, it is not necessarily a particular brand of cars whose consumption is advertised as a symbol of belonging to a certain status group that attracts increasing attention and expenditure shares. Rather it can be status seeking as such and its association with consumption as a proper way of symbolizing status that gains in importance relative to other to other needs that figure less prominently because there is less collective advertisement in their favor by other industries or no commercial advertisement at all.

Indeed, for the discussed deviation from conventional symbolic consumption to succeed, the unintended joint effect of individual advertizing activities which happen to coincide in emphasizing symbols that stand for the same need may be decisive. In terms of the model above, what may not be feasible for a single producer's advertising campaign may be achieved by the unplanned joint effect of a whole industry's campaign: the efficacy $\lambda$ and/or the cumulative effort $x(t)$ of placing information may be driven to levels so that the bias variable $b(t)$ approaches +1 and a groups' conservatism in symbolic consumption is overcome. If so, this finding would not support the hypothesis of a direct manipulation of consumer behavior, including symbolic consumption, by the producers' advertising. But the conjecture that advertising does have an effect on consumption would not be invalidated either. The slightly more complex explanation would be that the effect occurs where advertising resembles the provision of a public good that is not even intentionally produced.

\section{Conclusions}

In this paper the case of symbolic consumption has been discussed to demonstrate the relevance of understanding the motivational underpinnings of consumption decisions for explaining consumer behavior. Symbolic consumption is an important medium for expressing messages. In this respect, the motivation underlying the corresponding spending behavior differs from that underlying non-symbolic consumption in a significant way. The motivation is at least in part to gain recognition by the kind of consumption chosen, be it status recognition, recognition of belonging to, or being different from, targeted groups, or recognition of one's self-perception. Unlike other consumption, in all these cases symbolic consumption presupposes valid, approved symbols to serve its purpose. The actual technological characteristics of consumer products serving as symbols have therefore been claimed to be of secondary importance. What matters instead is whether a social convention exists as to what goods and services function as symbols and what they mean.

Furthermore, it has been claimed that for the spontaneous emergence of the convention two logically distinct (though factually often coinciding) conditions must be met. First, there must be some common understanding among the involved individuals as to what the message of symbolic consumption is. Second, on the basis of this common understanding, the individuals then have to coordinate on which symbols to use when. The first of the two conditions, the emergence of a shared understanding, has been shown to rest on a particular feature of human behavior. This feature is the capacity of a socially embedded cognitive learning, the dynamic correlate of bounded rationality. The second of the two conditions is contingent on a feature that all conventions share. Their strength depends on how widely the convention is approved in the actual interaction, in this case the signaling by means of symbolic consumption. It is precisely in this point that the tacit agreements on symbolic consumption that are spontaneously emerging 
within groups with intense internal communication can be challenged. If behavior deviating in the use of symbols occurs, the other group members are confronted with not previously recognized alternatives in symbolic consumption and with the choice of whether or not to imitate the deviation. This choice directly affects the stability of the existing conventional basis of symbolic consumption.

By a simple model of communication processes and agenda effects the stability conditions have been explored in more detail. As it turned out, the analyzed processes tend to conserve existing conventions so that conventional symbols, once they are established, tend to resist attempt to deviate and shifts to new symbols. These findings have suggested to ask what influence advertising can have on symbolic consumption. The tentative answer which the paper suggests is that there is little theoretical support for the hypothesis of a direct influence of some producer's advertising campaign on symbolic consumption. However, the conjecture that advertising does have an effect on consumption is not invalidated either. The slightly more complex explanation offered is that advertising develops an effect as the unplanned consequence of a competitive advertising race of an entire industry that, by the symbols propagated, happens to jointly emphasize the same need.

\section{References}

Anderson, J.R. (2000),

Cognitive Psychology and Its Implications. San Francisco: Worth Publishers, $4^{\text {th }}$ edit. Bandura, A. (1986)

Social Foundations of Thought and Action - A Social Cognitive Theory, Englewood Cliffs: Prentice-Hall.

Bourdieu, P. (1984)

Distinction, Cambridge, MA.: Harvard University Press.

Frank, R.H. (1985)

Choosing the Right Pond - Human Behavior and the Quest for Status, Oxford: Oxford University Press.

Frank, R. H. (1999),

Luxury Fever - Money and Happiness in an Era of Excess, Princeton: Princeton University Press.

Galbraith, J.K. (1973),

Economics and the Public Purpose. Boston: Houghton Mifflin.

Lorenz, H.-W. (1993),

Nonlinear Dynamical Economics and Chaotic Motions, $2^{\text {nd }}$ edition, Berlin: Springer.

Heffetz, O. (2009)

Symbolic Consumption in Economics: Applications and Implications, mimeo.

Helson, H. (1964),

Adaption Level Theory. New York: Harper and Row.

Hirsch, F. (1978)

Social Limits to Growth, Cambridge, MA.: Harvard University Press.

Lebergott,S. (1993)

Pursuing Happiness - American Consumers in the Twentieth Century, Princeton:

Princeton University Press. 
Leibenstein, H. (1950),

Bandwagon, Snob and Veblen Effects in the Theory of Consumers' Demand, Quarterly Journal of Economics, Vol. 65, 183-207.

Moore, H. (1994),

A Passion for Difference, Bloomington: Indiana University Press.

Nguyen, P., Saviotti, P.P., Trommeter, M., Bourgeois, B. (2005)

Variety and the Evolution of Refinery Processing. Industrial and Corporate Change, 14, 469-500.

Radner, R., Rothschild, M. (1975),

On Allocation of Effort. Journal of Economic Theory, Vol. 10, 358-376.

Saviotti, P.P. (1996)

Technological Evolution, Variety and the Economy. Aldershot: Edward Elgar.

Saviotti, P.P. (2001)

Variety, Growth and Demand. Journal of Evolutionary Economics, 11, 119-142.

Saviotti, P.P. and Metcalfe, J.S. (1984)

A Theoretical Approach to the Construction of Technological Output Indicators.

Research Policy, 13, 141-151.

Saviotti, P.P. and Trickett, A. (1992)

The Evolution of Helicopter Technology 1940-1986. Economics of Innovation and New Technology, 2. 111-130.

Simon, H.A. (1972),

Theories of Bounded Rationality, in C.B.McGuire, R.Radner (eds.), Decision and

Organization, Amsterdam: North-Holland, 161-176.

Sugden, R. (1989),

Spontaneous Order, Journal of Economic Perspectives, Vol. 3, 85-97.

Veblen, T. (1899),

The Theory of the Leisure Class, New York: MacMillan.

Weidlich, W. (2000),

Sociodynamics - A Systematic Approach to Mathametical Modeling in the Social

Sciences, Amsterdam: Harwood Acad. Publ.

Windrum, P. (2005)

Heterogeneous Preferences and New Innovation Cycles in Mature Industries: The

Amateur Camera Industry 1955-1974. Industrial and Corporate Change, 14, 1043-1074.

Witt, U. (2001)

Learning to Consume - A Theory of Wants and the Growth of Demand. Journal of

Evolutionary Economics, 11, 23-36. 


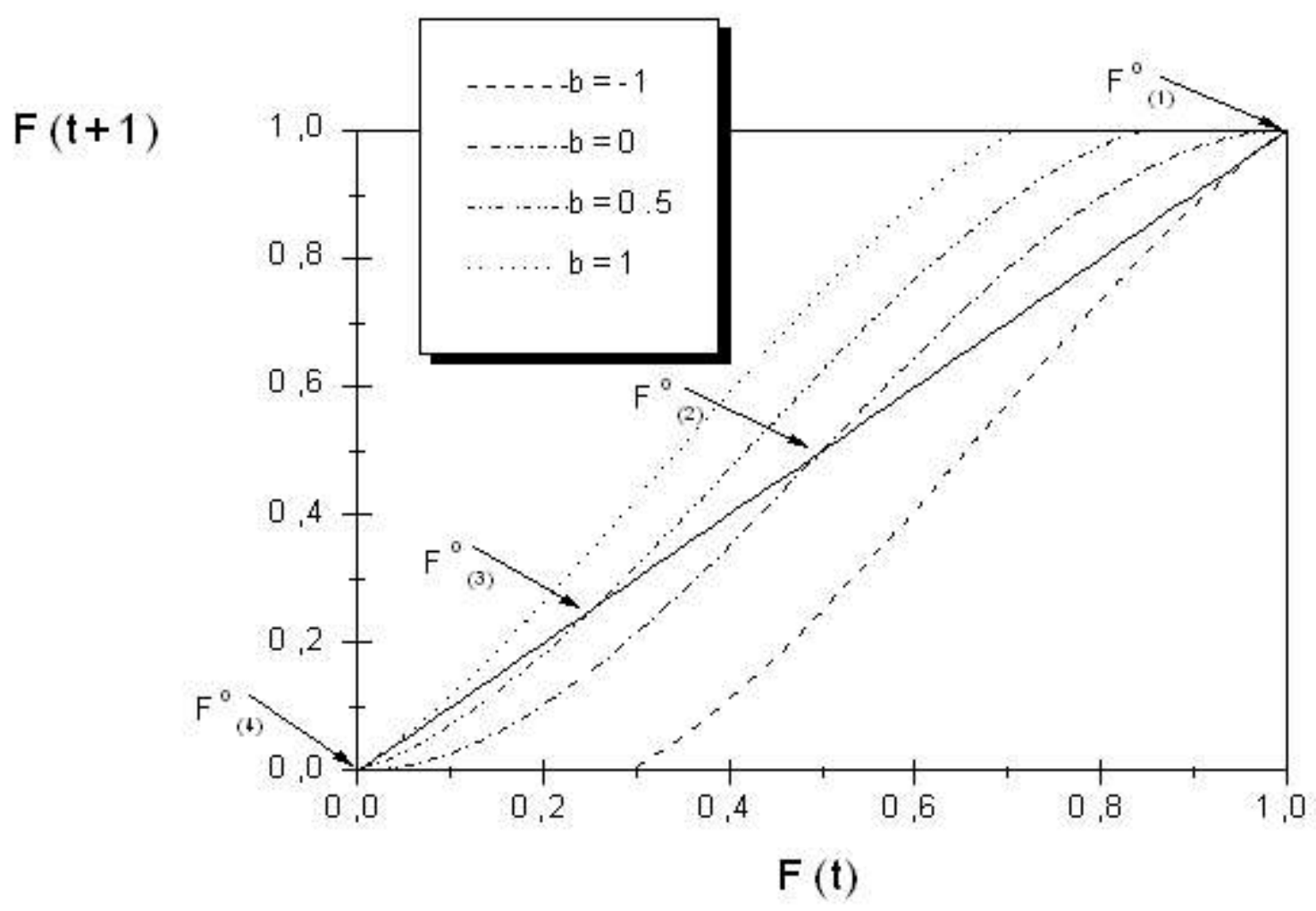

Figure 1: Bifurcation of Attractors in the Transition Between Conventions about Symbols 